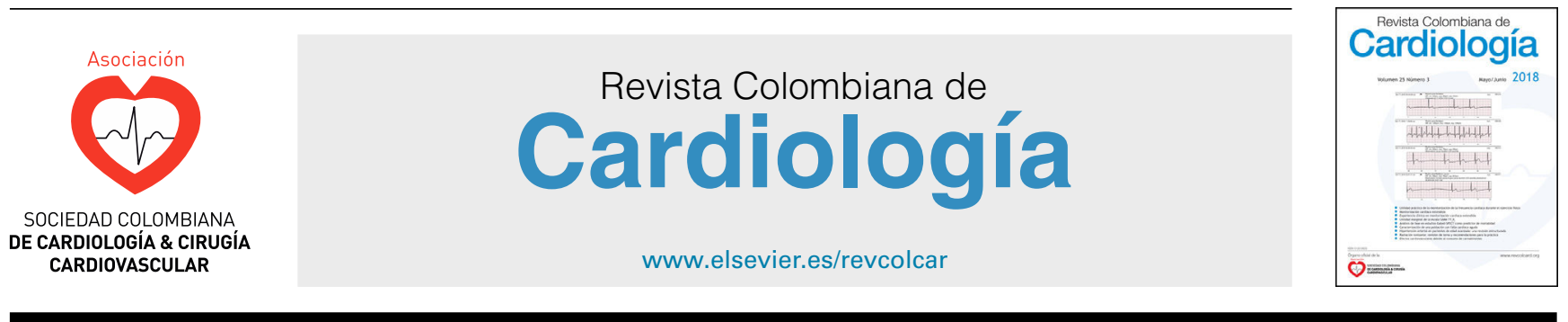

CARDIOLOGÍA DEL ADULTO - REVISIÓN DE TEMAS

\title{
Gestación y anticoagulación en válvula mecánica: un reto terapéutico
}

\section{Nelson L. Moreno Ruiz}

Grupo de Cardiología, Clínica Universitaria Colombia, Bogotá, Colombia

Recibido el 22 de agosto de 2017; aceptado el 26 de noviembre de 2017

Disponible en Internet el 7 de junio de 2018

\section{PALABRAS CLAVE \\ Prótesis valvulares \\ cardíacas; \\ Warfarina; \\ Heparina; \\ Heparina de bajo \\ peso molecular; \\ Embarazo}

\section{KEYWORDS}

Cardiac valve

replacement;

Warfarin;

Heparin;

Low molecular weight

heparin;

Pregnancy
Resumen La gestación en presencia de una válvula mecánica es un reto terapéutico tanto para el ginecólogo como para el cardiólogo, en vista de las complicaciones materno-fetales y mayor el riesgo de trombosis valvular. Medio siglo después del primer reporte de una gestación a término en este contexto, mucho se ha aprendido, pero persisten controversias importantes, de ahí que conocerlas sea fundamental para encontrar el tratamiento más balanceado.

(c) 2018 Sociedad Colombiana de Cardiología y Cirugía Cardiovascular. Publicado por Elsevier España, S.L.U. Este es un artículo Open Access bajo la licencia CC BY-NC-ND (http:// creativecommons.org/licenses/by-nc-nd/4.0/).

\section{Pregnancy and anticoagulation in mechanical valves: A therapeutic challenge}

Abstract Pregnancy and mechanical valves are a therapeutic challenge both for the gynaecologist and the cardiologist in view of the maternal-foetal complications, and the higher risk of valvular thrombosis. Half a century after the first report of a full term pregnancy in this context, much has been learned, but there are still significant controversies, which are essential to know about, in order to find the most balanced treatment.

(c) 2018 Sociedad Colombiana de Cardiología y Cirugía Cardiovascular. Published by Elsevier España, S.L.U. This is an open access article under the CC BY-NC-ND license (http:// creativecommons.org/licenses/by-nc-nd/4.0/).

\section{Introducción}

Ha pasado medio siglo desde la primera descripción de una gestación a término en una portadora de válvula mecánica ${ }^{1}$ y aunque se ha aprendido mucho desde entonces, continúan

Correo electrónico: neldoctor@yahoo.com las controversias y la variabilidad en los protocolos de manejo en estos complejos casos. Su asidero está en que por razones éticas, la realización de estudios clínicos de alta calidad metodológica, como se recomienda por la medicina basada en la evidencia, que comparen diferentes alternativas de anticoagulación no se han llevado a cabo y es poco probable que se hagan, así que los datos y recomendaciones que guían el actuar clínico se sustentan en estudios 
observacionales, de casos y controles, cohortes históricas y registros poblacionales, con las reconocidas limitaciones y sesgos que genera cada diseño. Por tanto, los metaanálisis derivados de estos estudios y las recomendaciones de expertos deben usarse con precaución y solo como una guía para orientar el manejo, que dependerá de diversas circunstancias.

\section{Qué se ha aprendido}

\section{Riesgo gestacional}

Diversos metaanálisis han coincido en mostrar que la mortalidad materna está entre el 1 a $4 \%^{2}$, lo cual puede ser casi 400 veces mayor que la presente en población sana ${ }^{3}$; la principal causa de esto es la trombosis valvular que se presenta hasta en el $5 \%$ de los casos. Además, la posibilidad de un evento adverso para la madre o el hijo es casi del $50 \%{ }^{4}$, hecho reconocido en diferentes modelos de riesgo gestacional como el ZAHARA y el OMS modificado $0^{5,6} \mathrm{n}$ las que se indica que tener una válvula mecánica ubica a esta gestante en grupos de alto riesgo y aunque esta condición no contraindica la gestación, algunos autores desaconsejan el embarazo si no es posible mantener una anticoagulación efectiva ${ }^{7}$. En Latinoamérica el trabajo pionero del grupo de Salazar et al. ${ }^{8}$ en su serie de 72 gestaciones, mostró una mortalidad materna del 4,3\% y de eventos trombóticos relacionado con la válvula de $11,4 \%$. El mismo grupo publicó en $2001^{9}$ su experiencia de más de tres décadas, con seguimiento de 304 gestantes portadoras de prótesis valvulares, en la que se halló una 1\% de mortalidad y 5,2\% de trombosis valvular. En 2001 se publicó en Colombia una serie de cinco casos de gestantes portadoras de válvulas mecánicas, en la que registraron dos muertes maternas asociadas al cambio de esquema de anticoagulación de warfarina a heparina no fraccionada subcutánea y heparina de bajo peso molecular, respectivamente ${ }^{10}$.

En registros recientes como el ROPAC (Registro de Gestación y Enfermedad Cardiaca de la Sociedad Europea de Cardiología) se encontró que la mortalidad materna fue similar entre los países desarrollados y los que están en vía de desarrollo (1 a 4\%), pero en los primeros hubo más admisiones hospitalarias y complicaciones hemorrágicas, explicadas en parte por el mayor número de cesáreas ${ }^{4}$.

\section{Riesgo del tratamiento con warfarina para el producto de la gestación}

Desde el primer reporte de la literatura quedo claro que la anticoagulación a causa de una válvula mecánica en una gestante, puede traer desenlaces diferentes tanto para la madre como para el producto ${ }^{1}$. En dicho reporte la gestante estuvo anticoagulada durante toda la gestación con warfarina y aunque su válvula mecánica no tuvo complicaciones, en el recién nacido hubo hallazgos teratogénicos que posteriormente se conocerían como embriopatía por cumarínicos.

Dado su peso molecular, la warfarina atraviesa la placenta y puede generar daño al producto de la gestación que se puede catalogar de la siguiente forma:

a. Embriopatía: se produce cuando se da entre el periodo de la organogénesis del primer trimestre. Se describen hallazgos como hipoplasia del puente nasal, obstrucción de coanas y condrodisplasia punteada. La patogenia se relaciona posiblemente con la alteración en la síntesis de la osteocalcina y la proteína de matriz Gla que modulan el depósito de calcio ${ }^{11,12}$.

b. Alteraciones en el sistema nervioso central: ocurren cuando se da más allá del primer trimestre. Se describen agenesia del cuerpo calloso, atrofia óptica y cerebral, ceguera, microcefalia, malformación tipo Dandy-Walker y bajo coeficiente intelectual ${ }^{13}$.

c. Efectos hemorrágicos: en la vida intrauterina hay disminución de los niveles de factores de coagulación vitamina $\mathrm{k}$ dependientes, inmadurez hepática y disminución de la unión de warfarina a la albúmina (desplazada por la bilirrubina) ${ }^{14}$, efectos que en conjunto aumentan el efecto anticoagulante de la warfarina en el feto. Este hecho explica que a pesar de un INR adecuado materno, el efecto fetal no se correlacione con este y la alta posibilidad de sangrados fetales, en especial si el parto es vaginal.

Salazar et al. ${ }^{8}$ reportaron efectos sobre el producto de la gestación casi hasta del $30 \%$ cuando se usó warfarina, bien desde el segundo trimestre o durante toda la gestación. Posteriormente, con el refinamiento y el análisis genético de los casos, en 2001 el mismo grupo 9 reportó un porcentaje de embriopatía del 4,1\%, el cual sería aún más bajo según los datos de los registros más recientes como el ROPAC, en el que sitúan la frecuencia de embriopatía y fetopatía en $2 \%$ y $1,4 \%$, respectivamente ${ }^{4}$.

Pero la warfarina no solo se asocia a efectos teratogénicos, fetopáticos o hemorrágicos, sino que las pérdidas gestacionales tempranas (aborto) y tardías (mortinato) son mayores que las reportadas con tratamientos basados en heparina ${ }^{2,4,9}$, lo cual concuerda en la mayoría de las series. En 1986 y posteriormente en 2001 Salazar et al. ${ }^{8,9}$ documentaron aborto hasta en el $26 \%$ de las gestaciones que usaron warfarina vs. $15 \%$ en aquellas que no lo hicieron. El registro ROPAC documentó que el uso de warfarina en el primer trimestre se asoció a aborto en $28 \%$ de los casos, casi tres veces mayor que la asociada al uso de heparina. Igualmente, el reporte de mortinatos fue del $7 \%$ para warfarina vs. $0,7 \%$ para heparina ${ }^{4}$.

\section{Riesgo del tratamiento con heparina para la madre}

La trombosis valvular es la principal complicación materna relacionada con una inadecuada anticoagulación con alta morbimortalidad $^{2,4}$. Dos metaanálisis publicados en 2017, concuerdan en que los protocolos con heparina no fraccionada (HNF) o de bajo peso molecular (HBPM), se asocian con mayor trombosis valvular (8\%) y mortalidad materna (5\%) que los tratamientos que usan warfarina en la gestación, bien sea durante toda la gestación con presencia de trombosis valvular del $2,7 \%$ y de mortalidad materna del $0,9 \%$ o como parte de tratamiento secuencial con heparina en el primer trimestre y warfarina en el segundo y tercer trimestre, con mortalidad materna del $2 \%$ y trombosis del $5 \%{ }^{15,16}$.

Los cambios fisiológicos de la gestación como aumento de la filtración glomerular, volumen sanguíneo y depuración renal, hacen que aumente la excreción de las HBPM, lo que 
modifica su farmacocinética y su efecto anticoagulante al compararse con un estado no grávido, de tal forma que es necesario monitorizar sus efectos a través de la actividad del factor $\mathrm{Xa}^{17}$. Estudios experimentales han mostrado que para mantener niveles terapéuticos de actividad del factor $\mathrm{Xa}$, a las 4 horas de aplicación de la heparina (niveles pico) durante la gestación, la dosis debe incrementarse casi un $54 \%$ (dosis final de $1,6 \mathrm{mg} / \mathrm{kg}$ cada 12 horas) cuando se compara con una dosis estándar fija de $1 \mathrm{mg} / \mathrm{kg}$ cada 12 horas $^{18}$. Igualmente, se ha mostrado que a las 8 horas de aplicación de la HBPM los niveles de actividad del factor Xa pueden ser subterapéuticos hasta en $20 \%$ de los pacientes, por lo que algunos proponen dividir la dosis cada 8 horas $^{19}$. Ciertos estudios que han usado HBPM durante la gestación, han mostrado trombosis valvular y mortalidad materna secundaria, a pesar de monitorizar los niveles pico de actividad $\mathrm{Xa}^{20}$. Esto llevó a una advertencia por parte de la FDA sobre el uso de esta medicación en anticoagulación de válvula mecánica en la gestación ${ }^{21}$. Una posible razón del porqué a pesar de la monitorización de la actividad pico del factor Xa se presenta trombosis valvular, proviene de estudios que muestran que no hay relación entres los niveles pico de actividad y los niveles valle (medidos 1 a 2 horas antes de la siguiente dosis), los cuales pueden ser inadecuados hasta en un $70 \%$ de los pacientes pese a niveles pico adecuados; por consiguiente podría ser necesario no solo monitorizar los niveles pico, sino también los valle ${ }^{22}$

Con base en esta evidencia la Sociedad Europea de Cardiología emite una recomendación en contra (recomendación III, nivel de evidencia $C$ ) del uso de heparina de bajo peso molecular en presencia de válvula mecánica en la gestación a menos que se monitorice la actividad del factor $\mathrm{Xa}^{6}$.

Con relación a la HNF, esta muestra una alteración en la biodisponibilidad durante el embarazo, de ahí que se necesiten mayores dosis y sea más difícil mantener el efecto anticoagulante cuando se administra por vía subcutánea; esto se debe al aumento en la unión a proteínas -factor de Von Willebrand-, alteraciones en el volumen plasmático, en la depuración renal y en la degradación placentaria de la heparina, por lo que pueden llegar a necesitarse dosis mayores a $35.000 \mathrm{U} / \mathrm{día}^{2,10}$. Salazar et al. ${ }^{9}$ reportaron que el uso de HNF subcutanéa se asoció con abortos en el $37 \%$ de los casos, hecho similar al hallado con warfarina y que se explica por la posibilidad de hemorragias en la unión uteroplacentaria. Lo anterior sumado a la posibilidad de osteoporosis "cuando se usa por más de 1 mes, y de desarrollar trombocitopenia inducida por heparina, hace que en la actualidad la heparina no fraccionada solo se use como terapia intravenosa por periodos cortos, bien sea durante el primer trimestre como parte de protocolos secuenciales o en el periodo periparto ${ }^{23}$.

\section{Áreas de controversia}

\section{Dosis bajas de warfarina - (menor o igual a $5 \mathrm{mg}$ )- vs. dosis alta de warfarina- $>5 \mathrm{mg}$ - y los efectos sobre el producto de la gestación}

En 1999, Vítale et al. ${ }^{24}$ sugirieron que los efectos nocivos de la warfarina sobre el producto de la gestación dependían de la dosis usada. En un registro de 58 gestaciones en 43 pacientes con válvulas mecánicas a los largo de 10 años se compararon aquellas gestaciones que usaron warfarina $5 \mathrm{mg}$ o menos al día durante toda la gestación (bajas dosis) con las que usaron warfarina a una dosis $>5 \mathrm{mg}$ al día (alta dosis) para lograr INR terapéutico. Se encontró ausencia de embriopatía, $12 \%$ de abortos y ningún mortinato en el grupo de bajas dosis vs. $8 \%$ de embriopatía, $72 \%$ de abortos y $4 \%$ de mortinatos en el grupo de alta dosis. El mismo grupo publicó tres años después ${ }^{25}$ otro estudio que mostró que la variable que se relacionaba con un desenlace gestacional adverso (aborto, mortinato, embriopatía o fetopatía) era el uso de warfarina a altas dosis ( $>5 \mathrm{mg}$ ), sin importar si era primigestante o multípara.

Estos estudios han sido la base para que se recomiende en las guías americanas y europeas (recomendación II, nivel de evidencia $A)^{6,26}$ el uso de warfarina a bajas dosis durante toda la gestación. Sin embargo, no todos los estudios han mostrado ausencia de riesgo con dosis bajas de warfarina. Pillay et al. ${ }^{27}$ en su estudio de 62 gestaciones en portadoras de válvula mecánica, encontraron embriopatía por warfarina en $5 \%$ de las madres expuestas durante el primer trimestre a este medicamento con una dosis diaria inferior o igual a $5 \mathrm{mg}$. Igualmente, el registro ROPAC no encontró diferencias en el número de abortos o mortinatos en pacientes que recibieron dosis altas o bajas ${ }^{4}$. Por tal razón los metaanálisis publicados recientemente hacen un llamado a ser cautos con el uso de warfarina (aún a bajas dosis) en la gestación, dado que no hay una dosis mínima libre de riesgos y que su uso en todos los casos debe hacerse luego de una asesoría adecuada y del consentimiento escrito de la paciente ${ }^{15,16}$.

\section{Monitorización pico y valle de la actividad factor Xa}

La monitorización de la actividad del factor Xa es imperativa si se desea usar HBPM. Dado que los niveles pico no se relacionan con los niveles valle, los cuales puede ser subterapéuticos hasta en $70 \%$ de los $\operatorname{casos}^{22}$ hecho que podría explicar los casos de trombosis valvular a pesar de niveles pico adecuados ${ }^{20}$, algunos expertos recomiendan la mediciones de niveles picos y valle de forma obligatoria cuando se usa HBPM y no solo los niveles pico ${ }^{22}$. Sin embargo, no hay estudios que comparen las dos estrategias de monitoreo por lo que la recomendación emitida por las sociedades científicas se limita a recomendar la medición de niveles pico $^{6,26}$.

\section{Protocolos de anticoagulación}

Se describen tres protocolos de anticoagulación en la actualidad que podrían ser usados:

a. Warfarina dosis baja ( $5 \mathrm{mg} /$ día) durante toda la gestación y cambio a heparina al término.

b. HBPM durante toda la gestación con medición obligatoria de actividad del factor Xa.

c. Secuencial: uso de heparina (HBPM o HNF IV) durante el primer trimestre, con posterior uso de warfarina durante el segundo y tercer trimestre y cambio a heparina al término. 
El protocolo de warfarina a dosis baja ( $5 \mathrm{mg} /$ día) busca reducir las complicaciones tromboembólicas maternas, minimizando la posibilidad de efectos adversos fetales, sin que en la actualidad exista una dosis mínima $100 \%$ segura para el producto de la gestación. Con este esquema el riesgo combinado de complicaciones materno-fetales está alrededor del $20 \%{ }^{16}$. En este esquema la warfarina se usa durante toda la gestación y solo en el momento del parto se cambia a heparina en infusión.

Un protocolo que solo usa HBPM durante todo el embarazo, busca primordialmente la seguridad del producto de la gestación, dado que al no cruzar la placenta no produce efectos teratogénicos. Sin embargo, el riesgo de complicaciones tromboembolicas es más alto, y existe posibilidad de muerte materna y fetal de forma secundaria, así que el riesgo combinado de complicaciones materno-fetales está alrededor del $25 \%{ }^{16}$. Como se ha mencionado previamente, es obligatoria la monitorización de actividad del factor Xa 4 horas luego de su aplicación -niveles pico- y sería ideal también monitorizar los niveles 1-2 horas antes de la siguiente aplicación -niveles valle $e^{22}$.

Un protocolo secuencial es aquel que usa heparina ya sea fraccionada intravenosa o HBPM durante el primer trimestre, con miras a evitar los riesgos teratogénicos, con posterior uso de warfarina durante el segundo y tercer trimestre para reducir así las complicaciones tromboembólicas. Se ha descrito que casi el $40 \%$ de los eventos de trombosis valvular se producen durante el periodo de transición de un esquema a otro ${ }^{28}$, tal como ocurrió en 2 de los 5 casos de la serie colombiana ${ }^{10}$, con un riesgo combinado de desenlaces adversos materno -fetales del $30 \%{ }^{16}$, de modo que se recomienda que se haga de forma intrahospitalaria. En países de bajos o medianos ingresos un protocolo secuencial podría balancear mejor tanto los riesgos maternos-fetales como las restricciones económicas, por lo que sería recomendable, como se sugiere en publicaciones recientes ${ }^{15}$, pese a que no existan estudios que comparen los diferentes protocolos en esos escenarios.

Sin embargo, en la actualidad no hay ningún protocolo que se asocie a un riesgo nulo para la madre o el producto de la gestación de forma simultánea y al no existir estudios que comparen estas diferentes modalidades, la elección depende de diversas variables (Ej. geográficas, económicas, de infraestructura del hospital) que hagan más o menos difícil el acceso a la monitorización de la anticoagulación. En este contexto (evidencia no concluyente a favor de alguna intervención) cobra importancia la percepción subjetiva que tanto la madre como el médico tengan sobre cuál riesgo es más aceptable (trombosis valvular vs. pérdida gestacional vs. embriopatía y/o fetopatía), lo cual puede estar influenciado por factores no medibles (experiencia previa del médico o la paciente, pérdidas gestacionales previas, temor del médico, temor de la madre) pero que inclinarán la balanza hacia algún protocolo en particular, efecto conocido como Rashomon ${ }^{29}$.

\section{Recomendación de las guías y los expertos}

En la figura 1se presenta la recomendación comparativa que da la Sociedad Europea de Cardiología (SEC) y la Asociación Americana del Corazón (AHA).

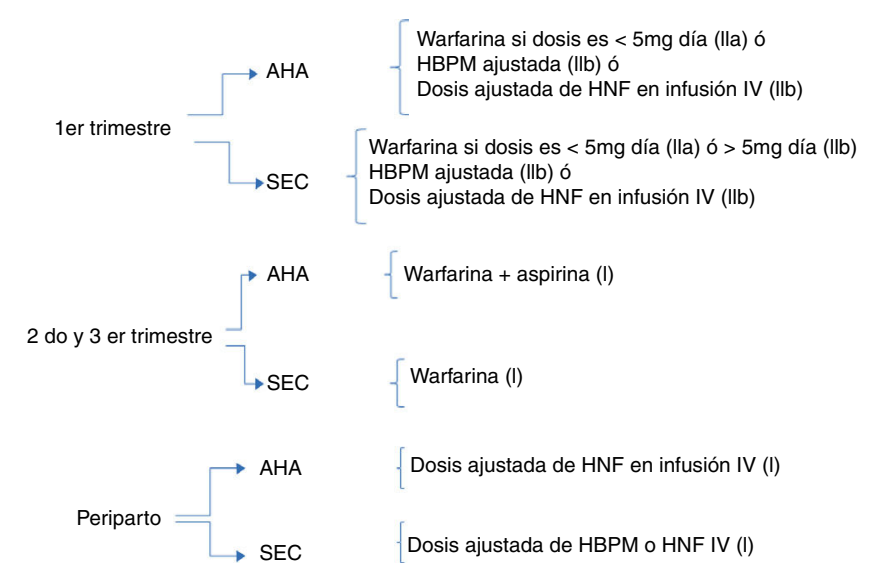

Figura 1 Recomendación Sociedad Europea de Cardiología y la Asociación Americana del Corazón sobre la anticoagulación en válvula mecánica durante la gestación.

Se destaca que la mayor posibilidad de variación está en los protocolos durante el primer trimestre. Ambas sociedades dan a las dosis bajas de warfarina una recomendación mayor (lla) que al uso de heparina no fraccionada o de bajo peso molecular (Ilb) durante el primer trimestre disminuyendo los desenlaces adversos maternos, pero como se ha mencionado previamente aún las dosis bajas de warfarina se han asociado a efectos embriopáticos, hecho que siempre deberá ser explicado de forma clara a la madre.

En el segundo y tercer trimestre hay acuerdo en el uso de warfarina asociado o no con aspirina, la cual podría usarse en condiciones de alto riesgo trombótico (ej: válvulas de primera-segunda generación, trombosis valvular previa, doble cambio valvular, válvulas en posición derecha).

En el periodo periparto es necesario tener en cuenta junto con la paciente y el grupo obstétrico variables como el modo de parto (cesárea o vaginal), el método de inducción y el uso de analgesia o anestesia regional. Si se planea la vía vaginal deberá suspenderse la warfarina en la semana 36 de gestación ${ }^{17}$ y reemplazarse con un protocolo de heparina (HNF o HBPM) con adecuada monitorización. La HBPM debería cambiarse a HNF al menos 12-24 horas antes del parto. La heparina no fraccionada deberá suspenderse 46 horas antes del parto y reiniciarse 4-6 posteriores si no hay complicaciones de sangrado. En caso de anticipación del trabajo de parto en una paciente bajo efectos con warfarina, se debe recordar que el feto está anticoagulado de ahí que existe riesgo de hemorragia intracraneal en caso de un parto vaginal y por tanto debería realizarse una cesárea electiva9.

Expertos como el Dr. Elkayam de la Universidad del Sur de California han implementado protocolos de anticoagulación basados en el riesgo trombótico valvular ${ }^{22}$ (fig. 2).

Se consideran pacientes de alto riesgo trombótico aquellas que tienen válvulas de primera generación en posición mitral, válvulas en posiciones derechas, historia de eventos trombóticos previos y fibrilación atrial. En estos casos se recomienda el uso de warfarina durante toda la gestación o un protocolo secuencial de heparina en el primer trimestre seguido de warfarina en el segundo y tercer trimestre ${ }^{30}$. Se consideran pacientes de bajo riesgo aquellas con válvulas 


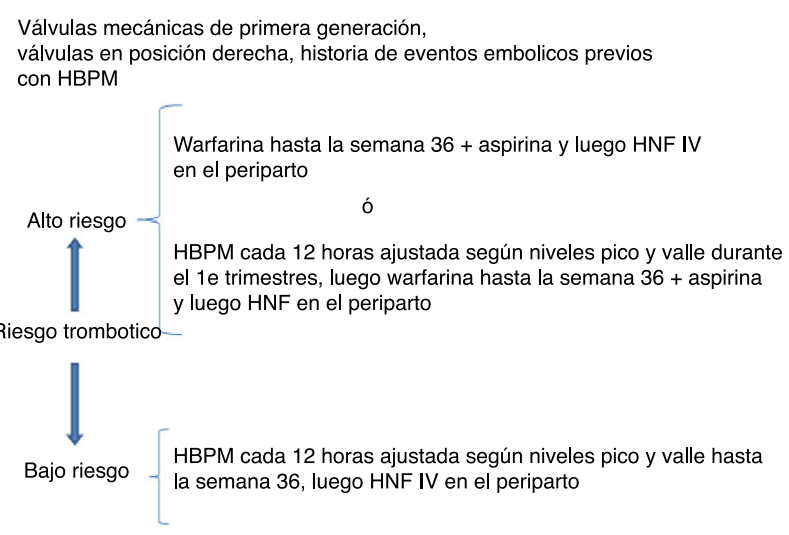

Válvulas mecánicas de nueva generación en posición aórtica y mitral

Figura 2 Protocolo de anticoagulación basado en el riesgo de trombosis valvular.

mecánicas de segunda y tercera generación en cualquier posición, en cuyos casos puede usarse heparina de bajo peso molecular durante toda la gestación, siempre y cuando se monitoricen los niveles de actividad pico y valle del factor $\mathrm{Xa}^{30}$.

Aunque esta aproximación resulta atractiva, en especial para los grupos de bajo riesgo, no ha sido estudiada y comparada con otros protocolos, enfatizando que es necesaria la medición estricta de los niveles pico y valle de la actividad del factor $\mathrm{Xa}$, hecho que por razones económicas puede no ser aplicable en todas las instituciones o en sitios de baja complejidad.

\section{Elección valvular}

La elección del mejor tipo de prótesis valvular en una mujer en edad reproductiva es compleja. Si bien las prótesis valvulares biológicas obvian la necesidad de anticoagular, estas tienen mayor riesgo de deterioro estructural, visto hasta en el $50 \%$ de las mujeres entre 16-39 años de edad al final de la primera década de la cirugía ${ }^{31}$ y que es mayor para las prótesis mitrales - siete veces más- que las aórticas o tricúspides $^{32}$. De otra parte, la necesidad de una reintervención puede tener un incremento de la mortalidad hasta del $9 \%{ }^{32}$. Una prótesis mecánica tiene la ventaja de una mayor durabilidad pero obliga a estar anticoagulado, lo cual adquiere más relevancia en el estado hipercoagulable del embarazo. Las prótesis de nueva generación (doble disco móvil) constituyen un gran avance ya que son menos trombogénicas que las iniciales y como se mencionó previamente en el protocolo del Dr. Elkayam ${ }^{30}$ podrían ser manejadas con heparina de bajo peso molecular siempre que se monitoricen los niveles pico y valle.

Una estrategia para orientar hacia la elección valvular, fue la propuesta por De Santo et al. ${ }^{33}$, en su estudio piloto con 22 mujeres en edad reproductiva que no estaban embarazadas y tenían valvulopatía aórtica no susceptible de reparación. Se realizó un protocolo de anticoagulación con warfarina y se evaluó quienes lograban un INR en metas (se eligió $1,5-2,5)$ con dosis bajas - $5 \mathrm{mg}$ o menos- y para quienes requerían dosis altas - más de $5 \mathrm{mg}$ día. En aquellas que se anticoagularon con dosis bajas (17 pacientes) se implantó una válvula mecánica y para aquellas que necesitaron dosis altas ( 5 pacientes) se propuso una válvula biológica. En las portadoras de válvula mecánica anticoaguladas con warfarina a bajas dosis no se registraron complicaciones materno-fetales en la gestación.

Este trabajo es pionero al intentar guiar la elección valvular en mujeres en edad reproductiva con base en la dosis de warfarina, pero solo se debe considerar como generador de hipótesis dado que únicamente aplicó para valvulopatía aórtica y además se escogió una meta de INR baja, que no es la recomendada en las guías de manejo de valvulopatía ${ }^{26}$.

\section{Conclusiones}

La gestación en presencia de una válvula mecánica, debe ser considerada siempre de alto riesgo y ser tratada de forma multidisciplinaria en instituciones de alto nivel de complejidad. No hay ningún régimen de tratamiento seguro $100 \%$ para la madre o el producto de la gestación de forma simultánea, aunque está claro que no se debe usar HBPM sin adecuada medición de los niveles de actividad del factor $\mathrm{Xa}$ en el pico (4 horas post aplicación) e idealmente también de niveles valle (1-2 horas antes de la siguiente dosis). Ante la ausencia de estudios comparativos entre los diferentes protocolos de anticoagulación, la elección que encuentre el mejor balance de riesgo materno-fetal, dependerá de variables económicas, geográficas, de infraestructura de las instituciones además de la subjetividad que tanto el médico como la paciente tengan sobre los beneficios y riesgos de cada uno.

\section{Conflicto de intereses}

Ninguno.

\section{Agradecimientos}

Al Grupo de Cardiología y Hemodinamia de la Clínica Colombia.

\section{Bibliografía}

1. Di Saia P. Pregnancy and delivery of a patient with a StarrEdwards mitral valve prosthesis: report of a case. Obstet Gynecol. 1966;28:469-72.

2. Chan W, Anand S, Ginsberg J. Anticoagulation of pregnant women with mechanical heart valves. Arch Intern Med. 2000;160:191-6.

3. Sillesen M, Hjortdal V, Vejlstrop N, Sorensen K. Pregnancy with prosthetic heart valves - 30 years' nationwide experience in Denmark. Eur Heart Cardio-Thorac Surg. 2011;40:448-54.

4. Hagen I, Hesselink J, Ruys T, Merz W, Goland S, Gabriel H, et al. Pregnancy in women with a mechanical heart valve. Data of the European Society of Cardiology Registry of Pregnancy and Cardiac Disease (ROPAC). Circulation. 2015;132:132-42.

5. Drenthen W, Boersma E, Balci A, Moons P, Hesselink J, Mulder $B$, et al. Predictors of pregnancy complications in women with congenital heart disease. Eur Heart J. 2010;31:2124-32.

6. Regitz-Zagrosev V, Blomstrom C, Borghi C, Ferreira R, Foidart J, Gibbs JS, et al. ESC Guidelines on the management of cardiovascular diseases during pregnancy. Eur Heart J. 2011;32:3147-97. 
7. Elkayam U, Goland S, Petronella P, Silversides C. High-risk cardiac disease in pregnancy. J Am Coll Cardiol. 2016;68:396-410.

8. Iturbe-Alessio I, Fonseca MC, Mutchinik O, Santos MA, Zajarías A, Salazar E, et al. Risks of anticoagulant therapy in pregnant women with artifical heart valves. N Engl J Med. 1986;27:1390-3.

9. Salazar E, Izaguirre R. Cardiopatía, anticoagulantes y embarazo. Rev Esp Cardiol. 2001;54 Supl 1:8-16.

10. Cardona C, Montoya J, Jaramillo C, Ospina C, Senior J. Anticoagulación en pacientes embarazadas, con prótesis valvular mecánica. Revisión de cinco casos y propuesta de tratamiento. Rev Col Cardiol. 2001;8:410-6.

11. Bates S, Ginsberg J. Anticoagulants in pregnancy: fetal effects. Clinical Obstetrics and Gynaecology. 1997;11:479-88.

12. Pauli RM. Mechanism of bone and cartilage maldevelopment in the warfarin embryopathy. Pathol Immunopathol Res. 1988;7:107-12.

13. James A, Brancazzio L, Ortel T. Thrombosis, thrombophilia, and thromboprophylaxis in pregnancy. Clinical Advances in Hematology \& Oncology. 2005;3:187-97.

14. Moalic P, Delahousse B, Body G, Bardis P, Leroy T, Gruel Y, et al. Evolution of Blood Coagulation Activators and Inhibitors in the Healthy Human Fetus. Blood. 1996;88:900-6.

15. DŚouza R, Ostro J, Shah P, Silverside C, Malinowski A, Murphy $\mathrm{K}$, et al. Anticoagulation for pregnant women with mechanical heart valves: a systematic review and meta-analysis. Eur Heart J. 2017;38:1509-16.

16. Steinberg Z, Dominguez-Islas C, Otto C, Stout K, Krieger E. Maternal and fetal outcomes of anticoagulation in pregnant women with mechanical heart valves. J Am Coll Cardiol. 2017;69:2681-91.

17. Castellano J, Narayan R, Vaishnava P, Fuster V. Anticoagulation during pregnancy in patients with a prosthetic heart valve. Nat Rev Cardiol. 2012;9:415-24.

18. Berresheim M, Wilkie J, Narenberg K, Ibrahim Q, Bungard T. A case series of LMWH use in pregnancy: Should trough anti-Xa levels guide dosing? Thrombosis Research. 2014;134:1234-40.

19. Friedrich E, Hameed AB. Fluctuations in anti-factor Xa levels with therapeutic enoxaparin anticoagulation in pregnancy. J Perinatol. 2010;30:253-7.

20. Yinon Y, Siu S, Warshafsky Ch, Maxwell C, McLeod A, Colman JM, et al. Use of low molecular weight heparin in pregnant women with mechanical heart valves. Am J Cardiol. 2009;104:1259-63.
21. Ginsberg J, Chan W, Bates S, Kaatz S. Anticoagulation of pregnant women with mechanical heart valves. Arch Intern Med. 2003;163:694-8.

22. Elkayam U. Anticoagulation therapy for pregnant women with mechanical prosthetic heart valves how to improve safety? J Am Coll Cardiol. 2017;69:2692-5.

23. Elkayam U, Bitar F. Valvular heart disease and pregnancy. J Am Col Cardiol. 2005;46:403-10.

24. Vitale N, De Feo M, De Santo L, Pollice A, Tedesco N, Cotrufo M, et al. Dose-dependent fetal complications of warfarin in pregnant women with mechanical heart valves. J Am Coll Cardiol. 1999;33:1637-41.

25. Cotrufo M, De Feo M, De Santo L, Romano G, Della Corte A, Renzulli A, et al. Risk of warfarin during pregnancy with mechanical valve prostheses. Obstet Gynecol. 2002;99:35-40.

26. Nishimura R, Otto C, Bonow R, Carabello R, Erwin J, Guyton RA, et al., 2014 AHA/ACC guideline for the management of patients with valvular heart disease: executive summary: a report of the American College of Cardiology/American Heart Association Task Force on Practice Guidelines. J Am Coll Cardiol. 2014;63:2438-88.

27. Pillay P, Nene Z, Mathiuha T, Macdonald A. The effect of warfarin dosage on maternal and fetal outcomes in pregnant women with prosthetic heart valves. Obstetric Medicine. 2011;4: 24-7.

28. Alshawabkeh L, Economy K, Valentine A. Anticoagulation during pregnancy. J Am Coll Cardiol. 2016;68:1804-13.

29. Heider K. The Rashomon effect: when ethnographers disagree. American Anthropologist. 1988;90:73-81.

30. Elkayam U, Singh H, Irani A, Akhter M. Anticoagulation in pregnant women with prosthetic heart valves. J Cardiovasc Pharmacol Ther. 2004;9:107.

31. Yun KL, Miller DC, Moore KA, Mitchell RS, Oyer PE, Stinson EB, et al. Durability of the Hancock MO bioprosthesis compared with standard aortic valve bioprostheses. Ann Thorac Surg. 1995;60:S221-8.

32. Jammiesson W, Rosado L, Monro A, Gerein A, Burr L, Miyagishima $\mathrm{R}$, et al. Carpentier-Edwards standard porcine bioprosthesis: primary tissue failure (structural valve deterioration) by age group. Ann Thorac Surg. 1998;46:155-62.

33. De santo L, Romero G, Corte A, Veronica D, Nappi G, Giordano $\mathrm{S}$, et al. mechanical aortic valve replacement in young women planning on pregnancy. J Am Coll Cardiol. 2012;59:12. 